\title{
Usefulness of Using Alternative Body-Mass Index and Neck Circumference Criteria for STOP-Bang Questionnaire in Screening South Korean Obstructive Sleep Apnea Patients
}

\author{
Jung-Ick Byun, MD, PhD ${ }^{1,2 *}$, Dong-Ha Kim, MD ${ }^{1 *}$, Jeong-Su Kim, MD¹, Won Chul Shin, MD, PhD ${ }^{1,2}$ \\ 'Department of Neurology, Kyung Hee University Hospital at Gangdong, Seoul, Korea \\ 2Department of Neurology, School of Medicine, Kyunghee University, Seoul, Korea
}

Received: May 23, 2020

Revised: June 11, 2020

Accepted: June 20, 2020

Correspondence

Won Chul Shin, MD, PhD

Department of Neurology,

Kyung Hee University at Gangdong,

892 Dongnam-ro, Gangdong-gu,

Seoul 05278, Korea

Tel +82-2-440-6166

Fax +82-2-440-7242

E-mail shin1chul@gmail.com

*These authors contributed equally to this work.

\section{ORCID}

Jung-Ick Byun

https://orcid.org/0000-0002-6224-4575

Dong-Ha Kim

https://orcid.org/0000-0003-1879-9976

Jeong-Su Kim

https://orcid.org/0000-0002-2892-0365

Won-Chul Shin

https://orcid.org/0000-0003-3044-9397

(C) This is an Open Access article distributed under the terms of the Creative Commons Attribution Non-Commercial License (https://creativecommons.org/licenses/by-nc/4.0) which permits unrestricted non-commercial use, distribution, and reproduction in any medium, provided the original work is properly cited.
Background and Objective The conventional criteria for body-mass index (BMI $>35 \mathrm{~kg} / \mathrm{m}^{2}$ ) and neck circumference $(\mathrm{NC}>40 \mathrm{~cm}$ ) in the snoring, tiredness, observed apnea, high blood pressure, BMI, age, NC, and gender (STOP-Bang) Questionnaire may be too high to screen Korean obstructive sleep apnea (OSA) patients properly. We hypothesized that using a modified STOP-Bang Questionnaire with lower BMI and NC criteria may be more appropriate when screening South Korean patients with OSA. We aimed to evaluate the utility of the modified STOP-Bang Questionnaires and to compare it with that of conventional STOP or STOP-Bang Questionnaire.

Methods This was a cross-sectional single-center study done at Kyung Hee University Hospital at Gangdong. Consecutive patients who underwent polysomnography and had done the STOPBang Questionnaire between November 2010 and December 2017 were reviewed. We used alternative cut-off criteria for BMI as $30 \mathrm{~kg} / \mathrm{m}^{2}$ and for $\mathrm{NC}$ as $36.3 \mathrm{~cm}$.

Results A total of 778 patients was evaluated; $73.1 \%$ of them had some OSA [apnea-hypopnea index $(\mathrm{AHI})>5 / \mathrm{hr}]$ and $54.5 \%$ had moderate to severe OSA (AHI $>15 / \mathrm{hr}$ ). The area under the curve (AUC) of the modified STOP-Bang was slightly larger than for the conventional STOP-Bang or STOP for having any OSA (AUC $0.787,0.781$, and 0.765 , respectively) or for moderate to severe OSA (AUC $0.749,0.747$, and 0.721). The conventional STOP-Bang Questionnaire with a cut-off score of 3 identified all OSA and moderate to severe OSA patients with a sensitivity of $94.7 \%$ and $96.9 \%$, respectively. The modified STOP-Bang showed improved sensitivity for any or moderate to severe OSA of $95.6 \%$ and $98.1 \%$, respectively, with a decrease in exchange for lower specificity.

Conclusions Because of the high prevalence of undiagnosed OSA, the modified STOP-Bang might be more appropriate in screening South Korean patients with OSA.

Sleep Med Res 2020;11(1):38-43

Key Words STOP-Bang Questionnaire, Obstructive sleep apnea, Body mass index.

\section{INTRODUCTION}

The STOP-Bang Questionnaire was first developed in 2008 and validated for screening patients for obstructive sleep apnea (OSA) surgery [1]. The questionnaire includes eight dichotomous items related to the clinical features of OSA: four subjective (STOP: snoring, tiredness, observed apnea, and high blood pressure) and four demographics items [Bang: body-mass index (BMI), age, neck circumference (NC), and gender]. The cut-off criteria of 3 for STOP-Bang is known to indicate a high risk of OSA, with sensitivity and specificity of $83.6 \%$ and $56.4 \%$, respectively [1]. Given its ease of use and high sensitivity, the STOP-Bang Questionnaire has been widely used in preoperative clinics, sleep clinics, the general population, and other special populations to detect patients at high risk of OSA [2]. 
A few studies evaluated the usefulness of the STOP-Bang Questionnaire for South Korean patients for screening OSA. One study showed that the STOP-Bang was the most useful tool among questionnaires, including the Berlin and Sleep Apnea of Sleep Disorder Questionnaire, with a sensitivity and specificity of $97.0 \%$ and $18.6 \%$ [3]. One recent study showed that the sensitivity and specificity of the STOP-Bang is $89.1 \%$ and $57.4 \%$, respectively, with an area under the curve (AUC) of 0.809 [4].

Obesity is a well-known risk for OSA [5]. Because the questionnaire was initially developed and validated in western countries, the conventional STOP-Bang Questionnaire used BMI > $35 \mathrm{~kg} / \mathrm{m}^{2}$ and $\mathrm{NC}>40 \mathrm{~cm}$ as cut-off criteria to describe obesity [1]. Criteria for obesity in Asians are different from those for Caucasians; so using the identical criteria has been questioned when using the STOP-Bang in screening Asian patients for OSA [6-8]. Moreover, Asian OSA patients are generally known to be less obese even with a high apnea-hypopnea index (AHI) [9]. The conventional criteria for the BMI and NC in the STOP-Bang may be too high to screen Korean OSA patients properly.

We hypothesized that using modified STOP-Bang Questionnaire with lower BMI and NC criteria may be more appropriate when screening South Korean patients for OSA. In this study, we evaluated the utility of the modified STOP-Bang and compared it to the conventional STOP or STOP-Bang Questionnaire.

\section{METHODS}

\section{Patients}

This was a cross-sectional single-center study done at Kyung Hee University Hospital at Gangdong. We reviewed consecutive adult patients who underwent polysomnography (PSG) between November 2010 and December 2017. Those who failed to complete the questionnaire or underwent the split-night PSG were excluded. This study was approved by the Institutional Review Board of the Kyung Hee University Hospital at Gangdong (IRB No. 2019-07-011). Informed consent to participate was obtained from the enrolled patients.

\section{Procedures}

For each patient, we reviewed demographics, results of sleep questionnaires, and PSG. Prior to the PSG, a patient's height, weight, and NC at the level of the cricoid cartilage was measured by the technician on duty.

An OSA diagnosis was made using standard PSG. The PSG was done using a digital polygraph system (Grass-Telefactor twin version 2.6, West Warwick, RI, USA) according to standard protocols. We recorded data from six electroencephalography channels (C3-A2, C4-A1, F3-A2, F4-A1, O3-A2, and O2A1) according to the standard $10-20$ system in addition to two electrooculography channels and surface electromyography of chin and both anterior tibialis muscles. We scored the data man- ually according to the American Academy of Sleep Medicine Manual for the Scoring of Sleep and Associated Events [10]. Apnea was defined as a drop in peak thermal sensor excursion by at least $90 \%$ of the baseline value for at least $10 \mathrm{~s}$. We defined hypopnea as a drop in the nasal pressure signal by at least $30 \%$ of the baseline value for at least $10 \mathrm{~s}$, with at least a $4 \%$ reduction in oxygen saturation from the pre-event baseline. The AHI was defined as the number of apnea and hypopnea events per hour. We based the diagnosis of any OSA on an AHI of 5 or more events per hour. Moderate to severe OSA was classified based on the AHI values $\geq 15 / h$.

\section{Modified STOP-Bang Using Alternative BMI and NC}

We used alternative BMI and NC criteria for the modified STOP-Bang. The alternative cut-off criteria for BMI and NC were set as follows: BMI as $30 \mathrm{~kg} / \mathrm{m}^{2}$ based on World Health Organization's definition of severe obesity in Asia-Pacific population [11], and NC as $36.3 \mathrm{~cm}$ based on the definition suggested by the Korean Obesity Society [12].

\section{Statistics}

Continuous data are presented as the mean \pm standard deviation. We used univariate and multivariate logistic regression analysis to evaluate the significance of each STOP-Bang and modified STOP-Bang Questionnaire components. We plotted receiver operating characteristics curves to assess the diagnostic accuracy of STOP, STOP-Bang, and modified STOP-Bang, using AHI cutoff points of 5 for any OSA and 15 for moderate to severe OSA. The greater AUC represents the better instruments. We calculated the sensitivity, specificity, positive predictive values, and negative predictive values of the STOP with a cut-off score of 2 , and the STOP-Bang and modified STOP-Bang with a cut-off score of 3 or 4 . The level of significance was set at $p<0.05$. All statistical comparisons were done with SPSS (version 22.0, IBM Corp., Armonk, NY, USA).

\section{RESULTS}

\section{Patient characteristics}

Among the 880 consecutive adult patients who underwent PSG between November 2010 and December 2017, we analyzed a total of 778 patients who had completed the questionnaire and underwent PSG between November 2010 and December 2017. Overall, the mean age was $49.3 \pm 13.3$ years, and $75.7 \%$ were male. Mean BMI and NC were $26.7 \pm 4.1 \mathrm{~kg} / \mathrm{m}^{2}$ and $37.8 \pm 3.9 \mathrm{~cm}$, respectively. The proportion of those with any OSA was $73.1 \%$, and $54.5 \%$ had moderate to severe OSA. Details of the sleep questionnaire and PSG findings are presented in Table 1.

Only 38 of the patients (4.9\%) met the criteria of BMI > 35 $\mathrm{kg} / \mathrm{m}^{2}$, and 139 (17.9\%) met the criteria of BMI $>30 \mathrm{~kg} / \mathrm{m}^{2}$. Prev- 
Table 1. Patient demographic, questionnaire score, and polysomnographic findings

\begin{tabular}{|c|c|}
\hline Age & $49.3 \pm 13.3$ \\
\hline Sex (male) & $589(75.7)$ \\
\hline Body-mass index $\left(\mathrm{kg} / \mathrm{m}^{2}\right)$ & $26.7 \pm 4.1$ \\
\hline$<18.5$ & $7(0.9)$ \\
\hline $18.5-22.9$ & $114(14.7)$ \\
\hline $23-24.9$ & $145(18.6)$ \\
\hline $25-29.9$ & $373(47.6)$ \\
\hline $30-34.9$ & $100(12.9)$ \\
\hline$\geq 35$ & $39(5.0)$ \\
\hline Neck circumference & $37.8 \pm 3.9$ \\
\hline \multicolumn{2}{|l|}{ Polysomnography finding } \\
\hline Total sleep time & $305.4 \pm 148.3$ \\
\hline N1 (\%) & $23.2 \pm 14.2$ \\
\hline N2 (\%) & $42.0 \pm 11.7$ \\
\hline N3 (\%) & $19.1 \pm 12.7$ \\
\hline REM (\%) & $15.7 \pm 7.7$ \\
\hline Wake after sleep onset (min) & $20.4 \pm 25.8$ \\
\hline Sleep efficacy (\%) & $80.6 \pm 12.1$ \\
\hline Arousal index & $46.7 \pm 22.2$ \\
\hline AHI & $27.6 \pm 39.0$ \\
\hline $\mathrm{AHI}<5 / \mathrm{hr}$ & $209(26.9)$ \\
\hline AHI 5-14.9/hr & $145(18.6)$ \\
\hline AHI $15-30 / \mathrm{hr}$ & $127(16.3)$ \\
\hline AHI $>30 / h r$ & $297(38.2)$ \\
\hline Lowest oxygen saturation & $80.2 \pm 11.2$ \\
\hline
\end{tabular}

Data are presented as $\mathrm{n}(\%)$ or mean \pm standard deviation. AHI: apnea-hypopnea index. alence of those who showed $\mathrm{NC}>40 \mathrm{~cm}$ was $182(23.4 \%)$, and 507 (65.2\%) had NC > $36.6 \mathrm{~cm}$ (Table 2).

\section{Significance of Each Component in Predicting Any or Moderate to Severe OSA}

The most significant factor predicting any OSA was observed apnea [odds ratio (OR) 7.84, $\mathrm{p}<0.001$ ] followed by BMI $>35$ $\mathrm{kg} / \mathrm{m}^{2}$ (OR 7.04, $\left.\mathrm{p}=0.008\right), \mathrm{NC}>40 \mathrm{~cm}(\mathrm{OR} 5.88, \mathrm{p}<0.001)$ and snoring (OR 5.43, $\mathrm{p}<0.001$ ), which was similar for predicting moderate to severe OSA. Tiredness and age $>50$ years old showed no significant value in predicting overall OSA or moderate to severe OSA. Although alternative criteria for BMI and $\mathrm{NC}\left(\mathrm{BMI}>30 \mathrm{~kg} / \mathrm{m}^{2}\right.$ and $\left.\mathrm{NC}>36.6 \mathrm{~cm}\right)$ showed lower OR than did the conventional criteria, they still showed significant power in predicting OSA or moderate to severe OSA (Table 2).

Multivariate logistic regression analysis showed that the observed apnea has the most significant independent predictive value for any or moderate to severe OSA in both the conventional and the modified STOP-Bang. Tiredness, BMI, or NC did not have an independent predictive value in the conventional STOPBang. In the modified STOP-Bang, the BMI and NC using the alternative criteria showed independent significance; on the other hand, age and gender did not show independent significance (Supplementary Table 1 in the online-only Data Supplement).

\section{Utility of the Modified STOP-Bang for Screening Any or Moderate to Severe OSA}

The AUC of the modified STOP-Bang was slightly larger than that of the conventional STOP-Bang or STOP for any OSA (AUC $0.787,0.781$, and 0.765 , respectively) or for moderate to severe OSA (AUC 0.749, 0.747, and 0.721) (Fig. 1, Table 3).

Using the conventional cut-off value of 3 , the sensitivity of the modified STOP-Bang for overall OSA was higher than that of the conventional one for any OSA (95.6\% vs. $94.72 \%$, respectively) or for moderate to severe OSA ( $98.11 \%$ vs. $96.93 \%$, respec-

Table 2. Individual logistic regression for STOP-Bang variables predicting OSA

\begin{tabular}{|c|c|c|c|c|c|c|c|}
\hline & \multirow{2}{*}{$\frac{\text { Total }(\mathrm{n}=778)}{\mathrm{n}(\%)}$} & \multicolumn{3}{|c|}{ Any OSA $(\mathrm{n}=568)$} & \multicolumn{3}{|c|}{ Moderate to severe OSA $(n=423)$} \\
\hline & & $\mathrm{n}(\%)$ & Exp (95\% range) & $\mathrm{p}$-value & $\mathrm{n}(\%)$ & Exp (95\% range) & p-value \\
\hline Snoring & $445(57.2)$ & $386(68.0)$ & $5.43(3.83-7.69)$ & $<0.001$ & $302(71.4)$ & $3.70(2.74-4.99)$ & $<0.001$ \\
\hline Tiredness & $696(89.5)$ & $508(89.4)$ & $0.99(0.59-1.66)$ & 0.972 & $380(89.8)$ & $1.09(0.69-1.73)$ & 0.711 \\
\hline Observed apnea & $587(75.4)$ & $492(86.6)$ & $7.84(5.45-11.27)$ & $<0.001$ & $380(89.8)$ & $6.32(4.32-9.23)$ & $<0.001$ \\
\hline Blood pressure & $270(34.7)$ & $226(39.8)$ & $2.49(1.72-3.62)$ & $<0.001$ & $185(43.7)$ & $2.47(1.81-3.37)$ & $<0.001$ \\
\hline $\mathrm{BMI}>30 \mathrm{~kg} / \mathrm{m}^{2}$ & $139(17.9)$ & $126(22.2)$ & $4.32(2.38-7.83)$ & $<0.001$ & $111(26.2)$ & $4.16(2.67-6.47)$ & $<0.001$ \\
\hline $\mathrm{BMI}>35 \mathrm{~kg} / \mathrm{m}^{2}$ & $38(4.9)$ & $36(6.3)$ & $7.04(1.68-29.49)$ & 0.008 & $33(7.8)$ & $5.92(2.29-15.34)$ & $<0.001$ \\
\hline $\mathrm{NC}>36.6 \mathrm{~cm}$ & $507(65.2)$ & $423(74.5)$ & $4.38(3.13-6.11)$ & $<0.001$ & $333(78.7)$ & $3.85(2.82-5.26)$ & $<0.001$ \\
\hline $\mathrm{NC}>40 \mathrm{~cm}$ & $182(23.4)$ & $168(29.6)$ & $5.88(3.32-10.41)$ & $<0.001$ & $147(34.8)$ & $4.87(3.26-7.28)$ & $<0.001$ \\
\hline Age $>50$ years & $384(49.4)$ & $278(48.9)$ & $0.94(0.69-1.29)$ & 0.704 & $203(48.0)$ & $0.89(0.67-1.18)$ & 0.405 \\
\hline Gender (male) & $589(75.7)$ & $467(82.2)$ & $3.34(2.35-4.73)$ & $<0.001$ & $357(84.4)$ & $2.87(2.04-4.04)$ & $<0.001$ \\
\hline
\end{tabular}

OSA: obstructive sleep apnea, BMI: body-mass index, Exp: exponent of the log odds, NC: neck circumference. 

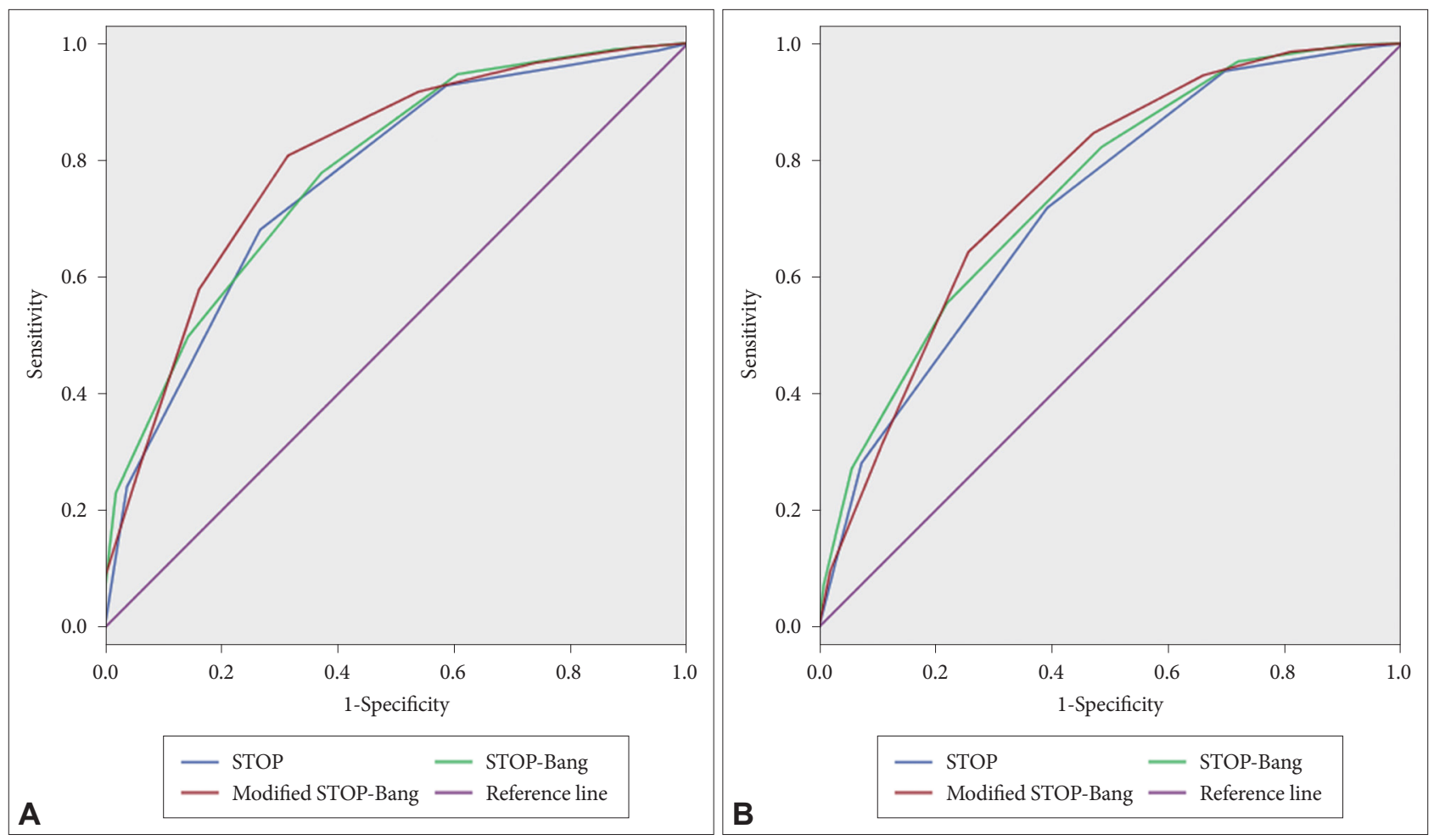

Fig. 1. Receiver operating curve for the three questionnaires compared to the reference line for $(A)$ any OSA and (B) moderate to severe OSA. OSA: obstructive sleep apnea, STOP-Bang: snoring, tiredness, observed apnea, high blood pressure, body-mass index, age, neck circumference, and gender.

Table 3. AUC for the STOP-Bang, modified STOP-Bang, and STOP

\begin{tabular}{lcc}
\hline & AHI $>5$ & AHI $>15$ \\
\hline STOP & $0.765(0.728-0.803)$ & $0.721(0.685-0.757)$ \\
STOP-Bang & $0.781(0.745-0.817)$ & $0.747(0.712-0.781)$ \\
Modified STOP-Bang & $0.787(0.750-0.824)$ & $0.749(0.715-0.783)$ \\
\hline
\end{tabular}

Data are presented as AUC value (95\% confidence interval).

AHI: apnea-hypopnea index, STOP-Bang: snoring, tiredness, observed apnea, high blood pressure, body-mass index, age, neck circumference, and gender, AUC: area under the curve.

tively), but with lower specificity. When we increased the cut-off score to 4 , the reduction in sensitivity was lower for the modified STOP-Bang and showed higher sensitivity than did the conventional STOP-Bang for any OSA ( $88.56 \%$ vs. $77.82 \%$, respectively) or for moderate to severe OSA ( $91.96 \%$ vs. $82.27 \%$, respectively), but with lower specificity. The STOP Questionnaire showed equivocal sensitivity and specificity than the STOP-Bang (Table 4).

\section{DISCUSSION}

We evaluated the usefulness of the modified STOP-Bang using alternative BMI and NC criteria for screening OSA in South Korean patients. Unlike previous Korean studies that evaluated the validity of the conventional STOP-Bang $[3,4]$, this study eval- uated the utility of the modified STOP-Bang with alternative BMI and NC criteria. The conventional STOP-Bang Questionnaire with a cut-off score of 3 identified any OSA and moderate to severe OSA patients with a sensitivity of $94.7 \%$ and $96.9 \%$, respectively. The modified STOP-Bang showed improved sensitivity for any or moderate to severe OSA to $95.6 \%$ and $98.1 \%$, respectively, with a decrease in exchange for lower specificity.

Observed apnea had the highest predictive value among the STOP-Bang items, which was in line with the previous study [4]. Tiredness was not a significant factor, because we included biased patients who visited sleep clinics and underwent PSG. They all had sleep-related symptoms, and $89.5 \%$ of them complained of tiredness. In the general population, only $39.7 \%$ of the participants answered "yes" to the tiredness component [8]. Symptoms related to OSA may be as important a predictor as are objective risk factors. The STOP Questionnaire, which mainly includes the symptom items, showed equivocal sensitivity and specificity compared to STOP-Bang.

The high cut-off criteria for BMI and NC has been an issue when using STOP-Bang in Asian populations. Mean BMI and $\mathrm{NC}$ in our patients were $26.7 \pm 4.1 \mathrm{~kg} / \mathrm{m}^{2}$ and $37.8 \pm 3.9 \mathrm{~cm}$, which were lower than those of the original Canadian cohort, with mean values of $30 \mathrm{~kg} / \mathrm{m}^{2}$ and $39 \mathrm{~cm}$. The proportion of patients exceeding BMI of $35 \mathrm{~kg} / \mathrm{m}^{2}$ was only $6.3 \%$, as in the previous study of South Koreans [12], which was lower than that of the Canadians (22.9\%) [13]. A lower proportion of patients may 
Table 4. STOP-Bang and modified STOP-Bang sensitivity, specificity for OSA

\begin{tabular}{|c|c|c|c|c|c|c|c|c|}
\hline & \multicolumn{4}{|c|}{ Any OSA } & \multicolumn{4}{|c|}{ Moderate to severe OSA } \\
\hline & Sens & Spe & PPV & NPV & Sens & Spe & PPV & NPV \\
\hline STOP $\geq 2$ & 92.78 & 41.43 & 81.08 & 67.97 & 95.27 & 30.42 & 62.00 & 84.38 \\
\hline STOP-Bang $\geq 3$ & 94.72 & 39.52 & 80.90 & 73.45 & 96.93 & 28.17 & 61.65 & 88.50 \\
\hline STOP-Bang $\geq 4$ & 77.82 & 62.86 & 85.00 & 51.16 & 82.27 & 51.55 & 66.92 & 70.93 \\
\hline Modified STOP-Bang $\geq 3$ & 95.60 & 31.43 & 73.01 & 79.04 & 98.11 & 23.38 & 60.41 & 91.21 \\
\hline Modified STOP-Bang $\geq 4$ & 88.56 & 55.24 & 84.25 & 64.09 & 91.96 & 41.41 & 65.16 & 81.22 \\
\hline
\end{tabular}

OSA: obstructive sleep apnea, Sens: sensitivity, Spe: specificity, PPV: positive predictive value, NPV: negative predictive value, STOP-Bang: snoring, tiredness, observed apnea, high blood pressure, body-mass index, age, neck circumference, and gender.

increase the specificity of the questionnaire, but may decrease its sensitivity. Because Asians have OSA despite lower AHI [14], lower BMI criteria were evaluated in several Asian studies.

Two studies done in Singapore concluded that lowering the BMI criterion does not change the performance of the STOPBang Questionnaire. One study evaluated in-lab patients and found that lowering the BMI criterion to $30 \mathrm{~kg} / \mathrm{m}^{2}$ increased the sensitivity in detecting moderate to severe OSA from $91.3 \%$ to 93.8\% without a decrease in specificity [6]. The other study evaluated the general population and found that the alternative BMI criterion with $30 \mathrm{~kg} / \mathrm{m}^{2}$ increased the sensitivity from $66.2 \%$ to $69.1 \%$, with decreased specificity from $74.7 \%$ to $73.0 \%$ [8]. The degree of change of the sensitivity and specificity with the modified BMI criterion was similar to our results; however, the increased AUC suggests that the modified STOP-Bang in our study may be better in screening South Korean OSA patients. A recent study in China used a BMI cut-off value of $28 \mathrm{~kg} / \mathrm{m}^{2}$, which increased the sensitivity of the modified STOP-Bang score $\geq 4$ in detecting moderate to severe OSA from $79.2 \%$ to $89.3 \%$ and reduced specificity from $43.6 \%$ to $38.2 \%$. Although they found no significant difference in sensitivity, lowering the BMI cut-off showed the highest Youden index [15].

$\mathrm{NC}$ is known to be an independent predictor of OSA and its severity in snoring South Korean patients [16]. We applied a lower NC criterion following the Korean Obesity Society [12], and this increased the independent OR associated with any or moderate to severe OSA. However, $23.4 \%$ of the patients met the NC $>40 \mathrm{~cm}$ criterion, and $65.2 \%$ of the patients showed $\mathrm{NC}>36.6$ $\mathrm{cm}$, which questions the validity of changing the NC criterion. A recent study showed that the optimal NC for predicting OSA in Singapore was $39 \mathrm{~cm}$ in males and $35 \mathrm{~cm}$ in females, which increased sensitivity from $71 \%$ to $83.4 \%$ and $32 \%$ to $84.6 \%$ respectively [17]. The NC criterion of $40 \mathrm{~cm}$ may be appropriate for screening OSA in South Korean males.

Although this study was a retrospective single-center study, it must be acknowledged that this work is the first to evaluate the feasibility of the alternative BMI and NC criteria in the STOPBang for Korean OSA patients. The true utility of the modified STOP-Bang should be validated in a general population.

The modified STOP-Bang using alternative criteria for BMI >
$30 \mathrm{~kg} / \mathrm{m}^{2}$ and $\mathrm{NC}>36.6 \mathrm{~cm}$ increased AUC and sensitivity in predicting any OSA or moderate to severe OSA but decreased its specificity. Because of the high prevalence of undiagnosed OSA, the modified STOP-Bang should be applied to screen South Koreans with higher sensitivity.

\section{Supplementary Materials}

The online-only Data Supplement is available with this article at https://doi.org/10.17241/smr.2020.00591.

\section{Acknowledgments}

This work was supported by a grant from Kyung Hee University in 2019 (KHU-20192274).

\section{Conflicts of Interest}

The authors have no financial conflicts of interest.

\section{Authors' Contribution}

Conceptualization: Byun JI, Shin WC. Data curation: Kim DH, Kim JS. Formal analysis: Byun JI. Investigation: Byun JI, Kim DH. Supervision: Shin WC. Writing—original draft: Byun JI, Kim DH. Writing—review \& editing: Shin WC.

\section{REFERENCES}

1. Chung F, Yegneswaran B, Liao P, Chung SA, Vairavanathan S, Islam S, et al. STOP Questionnaire: a tool to screen patients for obstructive sleep apnea. Anesthesiology 2008;108:812-21.

2. Chung F, Abdullah HR, Liao P. STOP-Bang Questionnaire: a practical approach to screen for obstructive sleep apnea. Chest 2016;149:631-8.

3. Kim B, Lee EM, Chung YS, Kim WS, Lee SA. The utility of three screening questionnaires for obstructive sleep apnea in a sleep clinic setting. Yonsei Med J 2015;56:684-90.

4. Kim KT, Cho YW. Real-world STOPBANG: how useful is STOPBANG for sleep clinics? Sleep Breath 2019;23:1219-26.

5. Peppard PE, Young T, Palta M, Dempsey J, Skatrud J. Longitudinal study of moderate weight change and sleep-disordered breathing. JAMA 2000;284:3015-21.

6. Ong TH, Raudha S, Fook-Chong S, Lew N, Hsu AA. Simplifying STOPBANG: use of a simple questionnaire to screen for OSA in an Asian population. Sleep Breath 2010;14:371-6.

7. Pavarangkul T, Jungtrakul T, Chaobangprom P, Nitiwatthana L, Jongkumchok W, Morrakotkhiew W, et al. The Stop-Bang Questionnaire as a Screening Tool for obstructive sleep apnea-induced hypertension in Asian population. Neurol Int 2016;8:6104.

8. Tan A, Yin JD, Tan LW, van Dam RM, Cheung YY, Lee CH. Predicting obstructive sleep apnea using the STOP-Bang Questionnaire in the general population. Sleep Med 2016;27-28:66-71. 
9. Lam B, Lam DC, Ip MS. Obstructive sleep apnoea in Asia. Int J Tuberc Lung Dis 2007;11:2-11.

10. Berry RB, Brooks R, Gamaldo CE, Harding SM, Lloyd RM, Marcus $\mathrm{CL}$, Vaughn BV. The AASM manual for the scoring of sleep and associated events: rules, terminology and technical specifications, version 2.2. Darien, IL: American Academy of Sleep Medicine, 2015.

11. WHO Expert Consultation. Appropriate body-mass index for Asian populations and its implications for policy and intervention strategies. Lancet 2004;363:157-63.

12. Kang JH, Ryu BY, Suh HS, Shim KW. Neck circumference as a simple obesity index. J Obes Metab Syndr 2002;11:142-9.

13. Chung F, Yang Y, Brown R, Liao P. Alternative scoring models of STOPBang Questionnaire improve specificity to detect undiagnosed obstruc- tive sleep apnea. J Clin Sleep Med 2014;10:951-8.

14. Young T, Shahar E, Nieto FJ, Redline S, Newman AB, Gottlieb DJ, et al. Predictors of sleep-disordered breathing in community-dwelling adults: the Sleep Heart Health Study. Arch Intern Med 2002;162:893-900.

15. Xia M, Liu S, Ji N, Xu J, Zhou Z, Tong J, et al. BMI $35 \mathrm{~kg} / \mathrm{m}^{2}$ does not fit everyone: a modified STOP-Bang Questionnaire for sleep apnea screening in the Chinese population. Sleep Breath 2018;22:1075-82.

16. Kim SE, Park BS, Park SH, Shin KJ, Ha SY, Park J, et al. Predictors for presence and severity of obstructive sleep apnea in snoring patients: significance of neck circumference. J Sleep Med 2015;12:34-8.

17. Loh JMR, Toh ST. Rethinking neck circumference in STOP-BANG for Asian OSA. Proc Singapore Healthc 2018;28:105-9. 
Supplementary Table 1. Multivariate logistic regression for STOP-Bang variables predicting OSA

\begin{tabular}{|c|c|c|c|c|c|c|}
\hline \multirow{2}{*}{ Criteria } & \multicolumn{2}{|c|}{ STOP } & \multicolumn{2}{|c|}{ STOP-Bang } & \multicolumn{2}{|c|}{ Modified STOP-Bang } \\
\hline & Exp (95\% range) & $\mathrm{p}$-value & Exp (95\% range) & $\mathrm{p}$-value & Exp (95\% range) & p-value \\
\hline \multicolumn{7}{|l|}{$\mathrm{AHI}>5$} \\
\hline Snoring & $3.20(2.17-4.72)$ & $<0.001$ & $2.95(1.98-4.40)$ & $<0.0001$ & $3.07(2.05-4.59)$ & $<0.001$ \\
\hline Tiredness & $0.68(0.37-1.24)$ & 0.209 & $0.71(0.38-1.31)$ & 0.275 & $0.65(0.35-1.21)$ & 0.176 \\
\hline Observed apnea & $5.15(3.45-7.68)$ & $<0.001$ & $4.32(2.84-6.57)$ & $<0.001$ & $4.29(2.82-6.55)$ & $<0.001$ \\
\hline Blood pressure & $2.46(1.62-3.73)$ & $<0.001$ & $2.11(1.35-3.29)$ & 0.001 & $1.96(1.25-3.06)$ & 0.003 \\
\hline $\mathrm{BMI}>35 \mathrm{~kg} / \mathrm{m}^{2}$ & & & $3.17(0.68-14.78)$ & 0.141 & & \\
\hline $\mathrm{NC}>40 \mathrm{~cm}$ & & & $1.36(0.91-2.06)$ & 0.138 & & \\
\hline Age $>50$ years & & & $2.70(1.43-5.10)$ & 0.002 & $1.52(1.00-2.30)$ & 0.050 \\
\hline Gender (male) & & & $1.74(1.13-2.68)$ & 0.012 & $0.99(0.57-1.71)$ & 0.964 \\
\hline $\mathrm{BMI}>30 \mathrm{~kg} / \mathrm{m}^{2}$ & & & & & $2.20(1.12-4.29)$ & 0.021 \\
\hline $\mathrm{NC}>36.6 \mathrm{~cm}$ & & & & & $2.76(1.66-4.60)$ & $<0.001$ \\
\hline \multicolumn{7}{|l|}{$\mathrm{AHI}>15$} \\
\hline Snoring & $2.35(1.69-3.29)$ & $<0.001$ & $2.10(1.48-2.96)$ & $<0.001$ & $2.19(1.55-3.09)$ & $<0.001$ \\
\hline Tiredness & $0.84(0.50-1.42)$ & 0.516 & $0.84(0.49-1.43)$ & 0.519 & $0.79(0.46-1.36)$ & 0.403 \\
\hline Observed apnea & $4.45(2.95-6.72)$ & $<0.001$ & $3.72(2.42-5.72)$ & $<0.001$ & $3.62(2.35-5.56)$ & $<0.001$ \\
\hline Blood pressure & $2.42(1.73-3.40)$ & $<0.001$ & $2.20(1.53-3.17)$ & $<0.001$ & $2.06(1.43-2.97)$ & $<0.001$ \\
\hline $\mathrm{BMI}>35 \mathrm{~kg} / \mathrm{m}^{2}$ & & & $3.01(1.05-8.61)$ & 0.040 & & \\
\hline $\mathrm{NC}>40 \mathrm{~cm}$ & & & $1.12(0.78-1.59)$ & 0.547 & & \\
\hline Age $>50$ years & & & $2.70(1.72-4.23)$ & $<0.001$ & $1.22(0.85-1.74)$ & 0.283 \\
\hline Gender (male) & & & $1.53(1.01-2.30)$ & 0.043 & $0.97(0.58-1.65)$ & 0.921 \\
\hline $\mathrm{BMI}>30 \mathrm{~kg} / \mathrm{m}^{2}$ & & & & & $2.43(1.49-3.98)$ & $<0.001$ \\
\hline $\mathrm{NC}>36.6 \mathrm{~cm}$ & & & & & $2.42(1.52-3.85)$ & $<0.001$ \\
\hline
\end{tabular}

STOP-Bang: snoring, tiredness, observed apnea, high blood pressure, BMI, age, neck circumference, and gender, OSA: obstructive sleep apnea, AHI: apnea-hypopnea index, BMI: body-mass index, NC: neck circumference, Exp: exponent of the log odds. 